have been of particular importance where the more liberal use of a scarce material in one country than in another is due, at least in part, to undeveloped technical processes and equipment. Recommendations made on this point in connexion with tungsten, tin, cobalt, mica, copper, zinc, graphite and asbestos have in some instances been reinforced by an advisory interchange of technicians. A special liaison has been set up to centralize the activities of the Board in a field covering a wide variety of commodities, and particularly to provide a defined channel for the exchange of information on matters of conservation.

Liaison has throughout been maintained with the parallel Combined Boards, and a Joint Advisory Committee has been set up with the Combined Food Board for questions relating to fertilizers. The Combined Production and Resources Board has been provided with a statement of the estimated supply to the end of 1943 for steel ingots and the key materials aluminium, rubber and copper, as well as with combined supply requirements summaries for some twenty-four materials actually or potentially in such short supply as to endanger any part of the production programme.

In regard to collaboration with others of the United Nations, through machinery already developed by the State Department and the Board of Economic Warfare in Washington and by the British Commonwealth Supply Council in London, as well as by direct discussions and negotiations, the resources of Central and South America, the Belgian and French overseas territories, the British Commonwealth and friendly neutrals have been and are being mobilized. For the U.S.S.R. requirements are covered in the Protocol Agreements in amounts which are adopted by the Board in any general allocations. Discussions have taken place between representatives of the U.S.S.R. and Britain and the United States with regard to the supply of certain materials from Russia.

With the passing of a greater range of materials into the category of "short or critical supply", it became necessary for the two countries closely to co-ordinate the planning of their export programmes involving such materials. A Combined Export Markets Committee was therefore set up under the auspices of the Board to watch over the export programmes of both countries for certain scarce materials, to ensure that the requirements of the importing countries are, so far as possible, met, but on a carefully controlled assessment of essentia] needs comparable to that obtaining in the United States and Great Britain. On this basis the Com mittee agrees upon joint export programmes in the light of the other factors involved-shipping, labour, political and financial considerations, maintenance of normal channels of trade, etc.

The report thus shows that the Board has been able to exercise its function of planning the raw materials side of the combined war effort in three principal ways. First, agreement has been secured, through the Board, between the two Governments concerned, to common action in regulating supply, distribution and use of the major strategical materials, with the object of ensuring that each country is put in a position to make its maximum contribution to the war effort on the production side. Secondly, by bringing the authorities of the two countries together in co-operative arrangements for purchasing and stipply, a scramble has been prevented for those 'secondary' materials which are, in their place, essential to the production programmes. Thirdly, the Board and its machinery have formed a centre for the discussion of day-to-day problems affecting either country, and for the compilation of information and evidence on which alone an effective solution for those problems can be based. The extent to which those facilities have been freely and fully used by the Governments concerned, and the fact that although the Board has no executive authority its recommendations have never been disregarded, offer the strongest justification for the hope that this machinery of joint control over world-wide resources will be continued and given wider scope to prevent a wild scramble for the diminished supplies after the War, frustrating our hopes for the most fruitful use of the world's resources in recovery.

\section{AIR-BLAST CIRCUIT-BREAKERS}

A PAPER read in London on March 4 before the Institution of Electrical Engineers by A. R. Blandford discusses notable improvements in circuitbreaker design with reference to the reduction in oil quantities and to its elimination.

The trend in the design of modern high-power circuit-breakers has always been in the direction of reducing the quantity of oil necessary for are interruption. A decided step in this direction was the introduction of the arc-control device which confined the are within an arcing chamber surrounding the contacts during arc interruption. The application of such devices, which usually take the form of a small container of liquid with restricted venting enclosed in the main containers of liquid, made possible a considerable reduction in oil quantity, particularly at the higher voltages. More recently, there has developed a strong demand for oil-less circuitbreakers.

Other forms of circuit-breakers using various extinguishing media have been developed, but only the two forms, oil and air-blast, have found general favour, and of these the growing popularity of the latter is accounted for by a proper appreciation of its superiority in principle of operation and behaviour. The inherent advantages in their order of merit are freedom from explosion, elimination of oilfire hazard, mechanical simplicity, suitability for duties requiring frequent operation, cleanliness of maintenance and operation, reduced maintenance, and facility for power closing.

The principle of the axial blast can be applied readily to voltages from $6 \cdot 6 \mathrm{kV}$. upwards at standard ratings. The question of susceptibility to rates of rise of recovery voltage can be overcome with ease by the incorporation of parallel resistors, which also limit harmful over-voltages. Air-blast breakers provide freedom from the dangers of explosion and they eliminate fire hazard completely. They are positive in operation and result in minimum arcing times at all values of current. They sustain a minimum deterioration of contacts, resulting in long life for repetitive operation. 'Making' contacts are exposed for ease of inspection. Clean and speedy inspection of arcing contacts is possible, only about three minutes being required to dismantle and inspect one interrupting chamber. Large-capacity batteries with their problems of regulation for closing purposes are eliminated. 